Report

\title{
Evaluation of cervical smears at King Hussein Medical Centre, Jordan, over three and a half years
}

\author{
S.R. Malkawi, ${ }^{1}$ R.M. Abu Hazeem, ${ }^{1}$ B.M. Hajjat ${ }^{1}$ and F.K. Hajjiri ${ }^{1}$
}

\begin{abstract}
SUMMARY Cervical smears taken from women referred for a check-up or with vaginal itching/discharge over a period of 3.5 years were reviewed at the King Hussein Medical Centre, Jordan. All smears were fixed with $96 \%$ alcohol, stained with Papanicolaou stain and screened microscopically. Of the smears from 1176 women aged $18-70$ years, $4.5 \%$ were classified as inadequate, $7.7 \%$ were normal and $79.9 \%$ showed nonspecific inflammation. Abnormal vaginal flora was found in $4.8 \%$ of cases, Candida albicans in $1.2 \%$, Trichomonas vaginalis in $0.9 \%$ and actinomycosis in 1 case. Dysphasic changes were rare: 9 cases $(0.8 \%)$ were classified as atypical squamous cells of undetermined significance (ASCUS) and 2 cases $(0.2 \%)$ were low-grade squamous intraepithelial lesion (LSIL). No cases of human papillomavirus infection (HPV) or cervical carcinoma were found.
\end{abstract}

\section{Introduction}

The cervical smear (Papanicolaou, Pap smear) is a routine screening test used for the detection of early cervical abnormalities, namely precancerous dysplastic changes of the uterine cervix [1], together with viral, bacterial, and fungal infections of the cervix and vagina. Cervical screening is a relatively simple, low cost and noninvasive method. Regular screening for cervical cancer reduces both the mortality and incidence of cervical carcinoma. Cervical neoplasia typically develops into invasive cancer over a 10-year period [3-6] and apparent cases of rapidly progressive cervical cancer are likely to be among women who have escaped screening and proper follow-up. Annual screening reduces the probability of developing invasive carcinoma by over $95 \%$ [2].
There is also epidemiological and experimental evidence that Pap smears are beneficial in detecting infections that are risk factors associated with cervical cancer, such as human papillomavirus (HPV) [7,8]. Societies where sexual activity starts at a young age and where multiple partners are common are at a higher risk of exposure to HPV than in conservative societies such as Jordan. HPV is a virus that infects reproducing cells, thus enhancing proliferation of the cell population; this increases the risk of transformation to high-grade lesions or carcinomas [9-11]. A cervical smear also detects vaginal infections such as Candida albicans, where patients present with physical discomfort, excess vaginal discharge, itching and other complaints.

In the absence of a national cervical screening programme in Jordan, the aim of

${ }^{1}$ Princess Iman Research and Laboratory Sciences Centre, King Hussein Medical Centre, Amman, Jordan.

Received: 21/05/03; accepted: 03/08/03

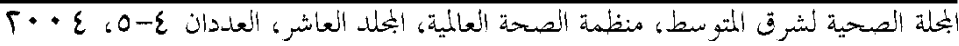


this study was to evaluate the prevalence of cervical lesions in cervical smears analysed at the Princess Iman Research and Laboratory Sciences Centre, Jordan.

\section{Methods}

Over a period of 3.5 years from August 1999 to February 2003, a retrospective review was made of records of 1176 cervical smears analysed at the Princess Iman Research and Laboratory Sciences Centre at King Hussein Medical Centre in Jordan. Patients were those who had been referred from all military hospitals in Jordan to the gynaecology clinic at the Centre with complaints of vaginal itching or discharge, and those who came for a first-time or followup cervical smear.

Cervical smears were taken by gynaecologists at the clinics using a speculum and brush; endocervical cells were smeared onto slides with direct fixation by $96 \%$ ethanol.

Smears were sent to the laboratory fixed in 96\% ethyl alcohol. All smears were stained with Papanicolaou stain and stained slides were screened microscopically by trained staff comprising 2 cytotechnologists and 1 pathologist. The adequacy of smears was determined by the presence of a good number of ecto- and endocervical components, no air dryness and no artefacts. All smears were routinely stained by Papanicolaou stain using a Leica Autostainer programmed for the purpose.

Slides were classified into 5 main categories: specific cervicitis, non-specific cervicitis, normal, cervical dysplasia, cervical carcinoma and inadequate.

\section{Results}

Of the cervical smears from 1176 women aged from 18-70 years, 91 (7.7\%) were normal, while 53 (4.5\%) smears were classified as inadequate (Table 1 ).

Of the remaining smears, 940 (79.9\%) showed non-specific inflammation, i.e. an inflammatory background with no evidence of viral changes or bacteria.

Specific inflammation was found in 81 cases: 56 (4.8\%) cases showed abnormal vaginal flora, including Gardnerella vaginalis, 14 cases had Candida albicans (1.2\%), 10 cases $(0.9 \%)$ had Trichomonas vaginalis and 1 case had actinomycosis $(0.1 \%)$. No cases of HPV infection were found.

Low-grade cervical abnormalities were seen in 11 cases: 9 cases $(0.8 \%)$ were classified as atypical squamous cells of undetermined significance (ASCUS) and 2 cases $(0.2 \%)$ were low-grade squamous intraepithelial lesion (LSIL). No malignant cases were reported within this study peri-

\begin{tabular}{lrc}
\hline Table 1 Classification of 1176 & cervical smears \\
\hline Category & $\begin{array}{c}\text { No. of } \\
\text { smears }\end{array}$ & $\%$ \\
\hline Inadequate & 53 & 4.5 \\
Normal & 91 & 7.7 \\
Non-specific cervicitis & 940 & 79.9 \\
Specific cervicitis & & \\
$\quad$ Abnormal vaginal flora, & & \\
including Gardnerella vaginalis & 56 & 4.8 \\
Candida albicans & 14 & 1.2 \\
$\quad$ Trichomonas vaginalis & 10 & 0.9 \\
$\quad$ Actinomycosis & 1 & 0.1 \\
Cervical dysplasia & & \\
$\quad$ ASCUS & 9 & 0.8 \\
$\quad$ LSIL & 2 & 0.2 \\
Cervical carcinoma & 0 & 0 \\
Total & 1176 & 100.0 \\
\hline
\end{tabular}

ASCUS = atypical squamous cells of undetermined significance.

$L S I L=$ low-grade squamous intraepithelial lesions .

لبحلة الصحية لشرق المثتوسط، منظمة الصحة العلمية، البحلد العاشر، العدذان ع-0، ع • • 
od. None of the categories were clustered in any specific age group.

\section{Discussion}

The cervical smear is a widely used routine test with many benefits, especially in detecting early cervical changes that can be treated to limit dysplastic processes developing into cancer. Of the cervical smear tests on 1176 women in our hospital, 79.9\% showed non-specific inflammation, namely unexplained inflammatory background, thus showing no bacterial or viral features. The remaining cases of inflammation showed $4.8 \%$ cases of specific inflammation, $1.2 \%$ candida infections, $0.9 \%$ trichomonal infections and 1 case of actinomycosis.

The incidence of dysplastic changes in our study $(1.0 \%)$ was low compared with other studies performed in industrialized countries $[1,13,14]$ and we found no cases of cervical carcinoma. This contrasts, for example, with a study in New England in the United States of America (USA) which found that $11.8 \%$ of women aged $20-29$ years and $8.4 \%$ of those over 30 years had infectious processes and $3.5 \%$ and $1.3 \%$ respectively showed squamous intraepithelial lesions (SIL) [15].

No cases of HPV infection were recorded in our hospital during this study period. Statistics from the Centers for Disease Control and Prevention's National Center for HIV, STD, and TB Prevention showed that 5.5 million people in the USA became infected with HPV each year, and infection rates were highest in young women [12]. In Jordan, sexual activity typically starts only after marriage where the marital age is over 16 years, and the cultural and religious traditions of our conservative society restrict the likelihood of multiple sexual partners. This may explain why no cases of sexually transmitted HPV, or cervical carcinoma, were found in our study group of women.

\section{References}

1. Greenlee RT et al. Cancer statistics, 2000. CA: a cancer journal for clinicians, 2000, 50(1):7-33.

2. McCrory DC et al. Evaluation of cervical cytology. Evidence report/technology assessment no.5. Rockville, Maryland, Agency for Health Care Policy and Research, 1999 (AHCPR publication no.99-E010).

3. Schwartz PE et al. Rapidly progressive cervical cancer: the Connecticut experience. American journal of obstetrics and gynecology, 1996, 175:1105-9.

4. Frame PS, Frame JS. Determinants of cancer screening frequency: the example of screening for cervical cancer. Journal of the American Board of Family Practice, 1998, 11:87-95.
5. Kenter GG et al. The cytological screening history of 469 patients with squamous cell carcinoma of the cervix uteri; does interval carcinoma exist? Acta obstetricia et gynecologica scandinavica, 1996, 75:400-3.

6. IARC Working Group on Evaluation of Cervical Cancer Screening Programmes. Screening for squamous cervical cancer: duration of low risk after negative results of cervical cytology and its implications for screening policies. British medical journal, 1986, 293:65964.

7. National Institutes of Health Consensus Development Conference statement on cervical cancer. April 1-3, 1996. Gynecologic oncology, 1997, 66:351-61. 
8. Schiffman MH, Bauer HM, Hoover RN. Epidemiologic evidence that human papillomavirus infection causes most cervical intraepithelial neoplasia. Journal of the National Cancer Institute, 1993, 85:958-64.

9. Stoler MH. A brief synopsis of the role of human papillomavirus in cervical carcinogenesis. American journal of obstetrics and gynecology, 1996, 175:1091-8.

10. Richart RM et al. Human papillomavirus, IAC Task Force summary. Acta cytologica, 1998, 42:50-8.

11. Stoler MH. Human papillomavirus and cervical neoplasia: a model for carcinogenesis. International journal of gynecological pathology, 2000, 19:16-28.

12. A closer look at HPV infection. In: Tracking the hidden epidemics 2000: trends in
STDs in the United States. Atlanta, Georgia, Centers for Disease Control and Prevention, 2000.

13. Johannesson G, Giersson G, Day N. The effect of mass screening in Iceland, 1965-74, on the incidence and mortality of cervical carcinoma. International journal of cancer, 1978, 21:418-25.

14. Hakama M et al. Effect of organized screening on the risk of cervical cancer in the Nordic countries. In: Miller AB et al., eds. Cancer screening. UICC project on evaluation of screening for cancer. Cambridge, United Kingdom, International Union Against Cancer, 1999:153-62.

15. Mount SL, Papillo JL. A study of 10,296 pediatric and adolescent Papanicoleau smear diagnosis in northern New England. Pediatrics, 1999, 103(3):539-45.

بلملة الصحية لشرق المثت سط، منظمة الصحة العالمية، البحلد العاشر، العلدان ع-0، ع + • 